\title{
Albumin-Fibrinogen ratio in rheumatoid arthritis and its relation to disease severity and radiographic progressive changes
}

\author{
Gihan O. Taher $^{1 *}$, Faiza A. Elhamdy ${ }^{2}$, Aisha M. Alfituri ${ }^{3}$, and Awad M. Alhasnony ${ }^{4}$ \\ ${ }^{1}$ Radiology Department, Faculty of Medicine, Tobruk University, Libya \\ ${ }^{2}$ Physiology Department, Faculty of Medicine, Benghazi University, Libya \\ ${ }^{3}$ Internal Medicine Department, Faculty of Medicine, Benghazi University, Libya \\ ${ }^{4}$ Biochemistry Department, Faculty of Medicine, Tobruk University, Libya \\ *Corresponding E-mail: gtaher@gmail.com
}

\begin{abstract}
Aim of the work: To evaluate the relationship between albumin-fibrinogen ratio (AFR) and radiographic changes in rheumatoid arthritis (RA). Patients and Methods: 50 rheumatoid patients and 30 control subjects were collected for the study, they were investigated for RF, Anti CCP, ESR, CR, fibrinogen, and albumin. Also, they were subjected to $x$-ray and MRI examination. Results: There was a significant increase in laboratory parameters and an increase in albumin fibrinogen ratio in rheumatoid patients as compared to healthy control subjects. Also, there was a positive correlation of the ratio with X-ray and MRI scores for synovitis and bone destruction. Conclusion: AFR may be easily, rapidly detected and prognostically useful markers of ongoing inflammation and joint affection correspondingly to radiological findings.
\end{abstract}

Keywords: rheumatoid arthritis, albumin-fibrinogen ratio, MRI, radiographic, plasma fibrinogen.

Received: 25 November, 2021

Accepted: 14 December, 2021

Published: 21 December, 2021

\section{Introduction}

Rheumatoid arthritis (RA) is a chronic inflammatory autoimmune disease of unknown etiology that affects mainly the joints [1]. Several cytokines are released lead to many inflammatory processes with final joint destruction [2].

Plasma fibrinogen expression and production by the liver may be increased during the inflammatory process. So, it is considered a positive acute-phase protein similar to erythrocyte sedimentation rate (ESR) and C-reactive protein (CRP) [3]. Meanwhile, serum albumin is expression may be decreased during the inflammatory process, unlike fibrinogen. So, it is categorized as a negative acute-phase protein [4]. Recently, a new concept of an inflammationrelated index using these two variables, fibrinogen to albumin ratio (FAR), has been introduced [5].

There is well-established evidence that albumin to fibrinogen ratio (AFR) is considered a potential biomarker reflecting the inflammatory status in malignant patients [6], in addition, it could be a possible predictor for clinical response to different cancer medications [7]. Also, some studies reported the adequate utility of it in expecting recanalization in myocardial infarction $[8,9]$. 
The aim of this work was to investigate the role of the albumin fibrinogen ratio in RA patients and to study their correlation with disease activity and joint severity.

\section{Patients and Methods:}

The study was carried out on 50 RA patients fulfilling the 2010 American College of Rheumatology (ACR)/European League against Rheumatism classification criteria for RA [10], and 30 healthy persons as control group matched in age and sex.

Conditions that are may change inflammatory markers' levels such as pregnancy, diabetes mellitus, hypertension, renal and/or hepatic dysfunction, malignancy, trauma or haemorrhagic events, and/or other coexisting connective tissue diseases were excluded from the study.

Informed written consents were obtained from the participants in this study. The study conforms to the 1995 Helsinki declaration and approved. The study protocol was treated confidentially corroborated to Helsinki declarations of biomedical ethics and approved by the research ethical committee of Benghazi Medical Centre Benghazi University.

All the patients were subjected to clinical assessment of disease activity of RA using the disease activity score in 28 joints (DAS 28-ESR) [11].

Laboratory investigations were done on patients and control groups: Erythrocyte sedimentation rate (ESR) in the first hour was estimated by Western method, serum CRP was quantified by immunoturbidimetry (Beckman Coulter) on AU 680 instrument. Serum albumin was measured by bromocresol green (Beckman Coulter) on AU 680. Plasma fibrinogen was quantified by Stago compact analyzer using the Clauss technique. Thus, Albumin to Fibrinogen (AFR) ratios was estimated

Plain x-ray in a standard posteroanterior (PA) view of the hands/wrists and anteroposterior (AP) view of the feet were taken and scored according to modified Larsen's score [12].

MRI was done to all patients and all the MRIs were scored for synovitis, bone oedema, and bone erosions, as defined in the Outcome Measures in Rheumatology (OMERACT) MRI rheumatoid arthritis recommendations [13].. By two radiologists who were blinded to the clinical findings.

\section{Statistical Analysis:}

After data collection and check for completeness and logical consistency. Pre-coded data were entered into Microsoft Office Excel 2010. Data were then transferred to Statistical Package for the Social Sciences, version 21. All statistical analyses were performed using two-tailed tests and an alpha error of 0.05 . A p-value distributed quantitative data, and frequencies categorical variables. Comparisons between groups were done using the MannWhitney test and Chi-square test. Spearman test was used for correlations.

\section{Results}

There was a significant increase in laboratory parameters and an increase in albumin fibrinogen ratio in rheumatoid patients as compared to healthy control subjects (table 1). Tables 2 demonstrate the radiographic score of rheumatoid patients. Also, there was a positive correlation of the AFR with X-ray and MRI score for synovitis and bone destruction (table 3).

Table 1: Demographics data and laboratory parameters in rheumatoid arthritis and control groups 


\begin{tabular}{|l|c|c|}
\hline & RA patients $(\mathbf{n}=\mathbf{5 0})$ & Controls(n=30) \\
\hline Age (years) & $45.35 \pm 6.76$ & $44.9 \pm 5.87$ \\
\hline Sex (male/female) & $2 / 48$ & $1 / 29$ \\
\hline Disease duration (years) & $10.50 \pm 43.9$ & --------- \\
\hline DAS-28 & $5.83 \pm 1.6$ & ---------- \\
\hline ESR (mm/1st h) & $38.85 \pm 18.5^{*}$ & $11.43 \pm 0.26$ \\
\hline CRP (mg/dl) & $25.22 \pm 3.6^{*}$ & $1.29 \pm 0.17$ \\
\hline RF (IU/ml) & $67.45 \pm 12.4^{*}$ & $5.60 \pm 1.16$ \\
\hline Anti-CCP(U/ml) & $233.6 \pm 67.6^{*}$ & $15.60 \pm 6.05$ \\
\hline Fibrinogen (g/l) Median & $5.23(1.01-4.15)$ & $1.45(1.23-1.10)$ \\
\hline Albumin (gm/dl) Median & $4(3.9-3.2)$ & $3.8(3.6-4.0)$ \\
\hline AFR Median (IQR) & $0.90(0.84-1.04)$ & $3(1.9-3.5)$ \\
\hline
\end{tabular}

Table 2: Radiological parameters in rheumatoid arthritis patients $(n=50)$

\begin{tabular}{|l|c|}
\hline Modified larsen score & $32.9 \pm 11.2$ \\
\hline MRI wrist joints scores & $4.4(1-7)$ \\
\hline Synovitis & $3.5(1-13)$ \\
\hline Bone erosions & $2.5(0-7)$ \\
\hline Bone defects & $1.6(0-11)$ \\
\hline Bone edema & \\
\hline MRI MCP joints scores & $6.9(2-10)$ \\
\hline Synovitis & $1.9(0-10)$ \\
\hline Bone erosions & $1.5(0-6)$ \\
\hline Bone defects & $1.4(0-19)$ \\
\hline Bone edema &
\end{tabular}

Values are mean (interquartile ranges) JSN, joint space narrowing; MCP, metacarpophalangeal.

Table 3: Correlation between AFR with clinical, laboratory, and radiological data in rheumatoid arthritis patients $(\mathbf{n}=\mathbf{5 0})$.

\begin{tabular}{|l|c|c|}
\hline \multirow{2}{*}{ Parameter } & \multicolumn{2}{|l|}{ AFR } \\
\cline { 2 - 3 } & $\mathbf{R}$ & $\mathbf{P}$ \\
\hline Age (years) & 0.271 & $\mathrm{NS}$ \\
\hline Disease duration (years) & 0.165 & $\mathrm{NS}$ \\
\hline DAS-28 & 0.819 & $<0.001 *$ \\
\hline ESR (mm/1 st hr) & 0.732 & $<0.001 *$ \\
\hline CRP (mg/dl) $\quad 0.675$ & $<0.001 *$ \\
\hline RF IU/ml) & 0.132 & $\mathrm{NS}$ \\
\hline Anti-CCP (U/ml) & 0.241 & $\mathrm{NS}$ \\
\hline Modified Larsen score & 0.763 & $<0.001 *$ \\
\hline MRI score for synovitis wrist joints & 0.522 & $<0.001 *$ \\
\hline
\end{tabular}




\begin{tabular}{|l|c|c|} 
MCP joints & 0.763 & $<0.001^{*}$ \\
\hline MRI score for erosion wrist joints & 0.861 & $<0.001^{*}$ \\
\hline MCP joints & 0.632 & $<0.001^{*}$ \\
\hline
\end{tabular}

*Pearson's correlation coefficient. NS: non-significant, ${ }^{*}=$ significantly different compared to controls. DAS-28, disease activity for 28 joint indices score; ESR, erythrocyte sedimentation rate; CRP, C reactive protein; RF, Rheumatoid factor; anti-CCP: anti-cyclic citrullinated peptide; mSvH: Modified Sharp/Van der Heijde, MRI: Magnetic resonance imaging, MCP: Metacarpophalangeal, AFR, albumin/fibrinogen.

\section{Discussion}

Rheumatoid arthritis is a progressive inflammatory musculoskeletal disease that causes systemic affection in addition to severe joint damage. To avoid its severe manifestations, early diagnosis and detection of disease activity is needed. Increased CRP, fibrinogen, and ferritin as acute phase reactants is a prominent feature of autoimmune diseases such as RA which reflects the inflammatory state $[14,15]$.

Our study revealed a significant increase in serum level of fibrinogen, ESR, and CRP in rheumatoid patients compared with the control group and this is usually occurring in inflammatory disorders [16].

Some previous studies demonstrated that AFR may be used as convenient laboratory markers, which indirectly reflect the state of inflammation and disease activity in some rheumatic diseases such as RA, AS [17].

In our study, there was a significant decrease in serum level of AFR in rheumatoid patients as compared to the control group and significantly negatively correlated with DAS, ESR, CRP and Larsen score and MRI scores, this is in agreement with previously reported by Yang et al who declared that AFR is correlated with DAS and CRP. Also, they could be simple predictors of disease activity in RA patients [17].

In our study, there was no relation between AFR ratio and RF\& Anti CCP, and this is in agreement with another study that revealed no relation between ratio and ACPA, but they are significantly correlated to RF titer [18].

In this study, it was found that AFR ratio are correlated with Larsen score and MRI score for synovitis and erosion These data suggest the strong association of fibrinogen and AFR with RA outcome and joint damage and these results are similar to another study [19].

Fibrinogen (FIB) is a key factor implicated in the process of the blood coagulation cascade, and its deposition in the joint was identified to be characteristic of RA and even may be responsible for the formation of pannus tissue [20].

FIB is an important glycoprotein present in human blood plasma and it is involved in many physiological processes such as wound healing, tissue regeneration, and regulation of inflammatory responses [26]. Moreover, FIB has been shown as an important determinant of inflammatory arthritis through its effects on pro-inflammatory pathways such as NF- $\mathrm{kB}$ signaling [21]. A recent study showed that circulating levels of FIB are elevated in RA and correlated with markers of inflammation [22] and this is in agreement with our study.

\section{Conclusion:}

AFR may be easily, rapidly detected and prognostically useful markers of ongoing inflammation and joint affection correspondingly to radiological findings. 


\section{DECLARATIONS}

\section{Funding}

This research did not receive any specific grant from funding agencies in the public, commercial, or not-for-profit sectors.

\section{Conflicts of Interest}

The authors declared no potential conflicts of interest with respect to the research, authorship, and/or publication of this article.

\section{REFERENCES}

[1] Chung SJ, Kwon YJ, Park MC, Park YB, Lee SKY. The correlation between increased serum concentrations of interleukin-6 family cytokines and disease activity in rheumatoid arthritis patients. Med J. 2011;52(1):113-120.

[2] Mervat EB. Sleep quality and fatigue in egyptian rheumatoid arthritis patients. Med J Cairo Univ. 2019;87(3):15571562. doi:10.21608/ mjcu.2019.53575

[3] Gabay C, Kushner I. Acute-phase proteins and other systemic responses to inflammation. N Engl J Med. 1999; 340(6): 448- 454.

[4] Tsirpanlis G, Bagos P, Ioannou D, et al. Serum albumin: a late-reacting negative acute-phase protein in clinically evident inflammation in dialysis patients. Nephrol Dial Transplant. 2005; 20(3): 658- 660.

[5] Tan Z, Zhang M, Han Q, et al. A novel blood tool of cancer prognosis in esophageal squamous cell carcinoma: the Fibrinogen/Albumin Ratio. J Cancer. 2017; 8(6): 1025- 1029.

[6] Sun D-W, Lin A, Guo Y. Albumin-fibrinogen ratio and fibrinogenprealbumin ratio as promising prognostic markers for cancers: an updated meta-analysis. World J Surg Onc. 2020; 18:9. doi:10.1186/ s12957-020-1786-2

[7] Lia S-Q, Jianga Y-H, Lin J, et al. Albumin-to- fibrinogen ratio as a promising biomarker to predict clinical outcome of non-small cell lung cancer individuals. Cancer Med. 2018;7(4):1221-1231. doi:10. 1002/cam4.1428

[8] Kayapinar O, Ozde C, Kaya A. Relationship between the reciprocal change in inflammation-related biomarkers (fibrinogen-to-albumin and hs-CRP-to-albumin ratios) and the presence and severity of coronary slow flow. Clin Appl Thromb Hemost. 2019; 25:10

[9] Yang WM, Zhang WH, Ying HQ, et al. Two new inflammatory markers associated with disease activity score-28 in patients with rheumatoid arthritis: albumin to fibrinogen ratio and C-reactive protein to albumin ratio. Int Immunopharmacol. 2018; 62:293-298. doi:10.1016/j.intimp.2018.07.007

[10] Aletaha D, Neogi T, Silman AJ, et al. Rheumatoid arthritis classification criteria: an American College of Rheumatology/European League Against Rheumatism collaborative initiative. Arthritis Rheum. 2010;62(9):2569 2581. doi:10.1002/art.27584

[11] 4. Prevoo m L, Van 'T Hof MA, Kuper HH. Modified disease activity scores that include twenty-eight-joint counts. Development and validation in a prospective longitudinal study of patients with rheumatoid arthritis. Arthritis Rheum. 1995;38(1):44-48. doi:10.1002/art.1780380107

[12] Rau R, Herborn G (1995) A modified version of Larsen's scoring method to assess radiologic changes in rheumatoid arthritis. J Rheumatol 22(10):1976- 1982

[13] Østergaard M, Peterfy C, Conaghan P, McQueen F, Bird P, Ejbjerg B, et al. OMERACT rheumatoid arthritis magnetic resonance imaging studies: core set of MRI acquisitions, joint pathology definitions, and the OMERACT RA-MRI scoring system. J Rheumatol 2003; 30(6):1385-1386.

[14] Singh HV, Shrivastava AK, Raizada A, et al. Atherogenic lipid profile and high sensitive C-reactive protein in patients with rheumatoid arthritis. Clin Biochem. 2013; 46:1007-1012. doi:10.1016/j. clinbiochem.2013.03.023

[15] Milman N, Karsh J, Booth RA. Correlation of a multi-cytokine panel with clinical disease activity in patients with rheumatoid arthritis. Clin Biochem. 2010; 43:1309—14.32. doi:10.1016/j.clinbiochem.2010.07.012

[16] Göbel K, Eichler S, Wiendl H, Chavakis T, Kleinschnitz C, Meuth SG. The coagulation factors fibrinogen, thrombin, and factor xii in inflammatory disorders - a systematic review. Front Immunol. 9:1731. 
doi:10.3389/fimmu.2018.01731

[17] Yang W-M, Zhang W-H, Ying H-Q, et al. Two new inflammatory markers associated with disease activity score-28 in patients with rheumatoid arthritis: albumin to fibrinogen ratio and C-reactive protein to albumin ratio. Int Immunopharmacol. 2018; 62: 293- 298.

[18] Kayapinar O, Ozde C, Kaya A. Relationship between the reciprocal change in inflammation-related biomarkers (fibrinogen-to-albumin and hs-CRP-to-albumin ratios) and the presence and severity of coronary slow flow. Clin Appl Thromb Hemost. 2019; 25:10

[19] Hameed B, Pilcher J, Heron C, Kiely PDW. The relation between composite ultrasound measures and the DAS28 score, its components, and acute phase markers in adult RA. Rheumatology. 2008;47(4):476-480

[20] So, A. K. et al. Arthritis is linked to local and systemic activation of coagulation and fibrinolysis pathways. J. Thromb. Haemost. JTH 1, 2510-2515. (2003).

[21] Liu, X. \& Piela-Smith, T. H. Fibrin (ogen)-induced expression of ICAM-1 and chemokines in human synovial fibroblasts. J. Immunol. 165, 5255 (2000).

[22] Rooney, T. et al. Levels of plasma fibrinogen are elevated in well-controlled rheumatoid arthritis. Rheumatology 50 , 1458-1465. (2011). 